\title{
Vibrational energy relaxation rate constants from linear response theory
}

\author{
Qiang Shi and Eitan Geva \\ Department of Chemistry and The FOCUS Center, University of Michigan, Ann Arbor, Michigan 48109-1055
}

(Received 22 October 2002; accepted 29 January 2003)

\begin{abstract}
A new approach for the calculation of vibrational energy relaxation rate constants is introduced. The new approach is based on linear response theory, and is shown to have several distinct advantages over the standard Landau-Teller formula, which is based on the Bloch-Redfield theory, namely: (1) weak system-bath coupling is not assumed; (2) selectivity in choosing the vibrational energy relaxation pathway, including non-Landau-Teller pathways, is possible; (3) the validity of rate kinetics can be explicitly verified; (4) direct extraction of the high-frequency tail of the force-force correlation function is avoided. A detailed analysis of the conditions under which the new expression reduces into the Landau-Teller formula, and an application in the case of bilinear coupling to a harmonic bath are provided. (C) 2003 American Institute of Physics.
\end{abstract}

[DOI: $10.1063 / 1.1562611]$

\section{INTRODUCTION}

One of the most fundamental ways in which the environment affects solution-phase molecular dynamics (MD) is via vibrational relaxation. A typical situation involves energy relaxation of an excited vibrational mode, in a solute molecule, by energy transfer to other intermolecular and/or intramolecular accepting modes. ${ }^{1-10}$ The rate of vibrational energy relaxation (VER) provides a sensitive probe of intramolecular dynamics and solute-solvent interactions, which are known to have a crucial impact on other important properties, such as chemical reactivity, solvation dynamics, and transport coefficients.

VER rates have been measured by time-domain pulsed laser techniques in a variety of hosts, including crystals, liquids, supercritical fluids, glasses, and proteins. ${ }^{9-39}$ The main experimental observations can be summarized as follows:

(1) In most cases, VER can be characterized by a single rate constant;

(2) VER can occur on a wide range of time scales, extending from subpicoseconds to minutes;

(3) VER can take place via a rich variety of intermolecular and/or intramolecular pathways.

The calculation of VER rate constants has presented theoretical chemistry with an ongoing challenge. Early studies of VER were based on the independent binary collision (IBC) model,,${ }^{17,40-44}$ which is based on the assumption that VER takes place via isolated and uncorrelated collisions with solvent atoms. However, the questionable validity of this assumption in the condensed phase $\mathrm{e}^{45-47}$ has led to alternative approaches, which rely on detailed MD simulations. Most studies have been based on either one of the following approaches: (1) The direct approach, which is based on nonequilibrium MD simulations; (2) The perturbative approach, which is based on extracting the force-force correlation function from equilibrium MD simulations.

The direct approach essentially mimics the experimental measurement of VER. It is based on performing nonequilibrium classical MD simulations, starting with an excited vibrational mode, and following its relaxation to equilibrium. ${ }^{48-52}$ This approach is particularly useful in the case of low-frequency vibrational modes and/or high temperatures $\left(\hbar \omega / k_{B} T \ll 1\right)$, since: (1) A classical description of the relaxing vibrational mode and the relevant accepting modes is permissible when $\hbar \omega / k_{B} T \ll 1$; (2) In these cases, VER is fast due to the high density of accepting modes with matching frequencies, and can therefore be directly observed on the time scales accessible to classical MD simulations.

Unfortunately, low-frequency vibrations are the exception rather than the rule, and most molecular vibrations are characterized by high frequencies, such that $\hbar \omega / k_{B} T \gg 1$ even at room temperature. This situation has two important implications:

(1) VER can become very slow, due to the very low density of accepting modes with matching frequencies, and therefore cannot be simulated on the time scale accessible to classical MD simulations (e.g., all neat diatomic liquids exhibit VER lifetimes of microseconds or longer ${ }^{10}$ ).

(2) A classical description of the relaxing vibrational mode and relevant accepting modes becomes inappropriate, and has to be replaced by a consistent quantum treatment of both.

The case of a harmonic mode bilinearly coupled to a harmonic bath, where the classical and quantum VER rate constants are identical, ${ }^{53}$ represents an important exception to the second statement above. However, this rather unique result is based on the balancing out of two opposing quantum effects, and relies on the fact that the coupling is bilinear. It has been found that the introduction of anharmonicities, in the coupling and/or the bath distorts this balance, and lead to deviations by orders of magnitude at high frequencies. ${ }^{54-56}$

The perturbative approach, which is outlined below, 
provides an alternative framework for the calculation of VER rate constants of such high-frequency vibrations. ${ }^{1,10,45,57}$ Its starting point is based on the following general quantum mechanical Hamiltonian of a harmonic vibrational mode coupled to a bath of accepting modes:

$$
\hat{H}=\hat{H}_{s}+\hat{H}_{b}+\hat{H}_{b s} \equiv \hat{H}_{0}+\hat{H}_{b s},
$$

where

$$
\hat{H}_{s}=\frac{\hat{p}^{2}}{2 \mu}+\frac{1}{2} \mu \omega^{2} \hat{q}^{2}
$$

is the Hamiltonian of the vibrational mode under investigation $(\hat{q}, \hat{p}, \mu$, and $\omega$ are the corresponding coordinate, momentum, reduced mass, and frequency, respectively),

$$
\hat{H}_{b}=\sum_{i=1}^{N} \frac{\left(\hat{P}^{(i)}\right)^{2}}{2 M^{(i)}}+\hat{V}\left(\hat{Q}^{(1)}, \ldots, \hat{Q}^{(N)}\right)
$$

is the Hamiltonian of the bath, which consists of the other intermolecular and intramolecular degrees of freedom $\left[\left\{\hat{Q}^{(i)}\right\},\left\{\hat{P}^{(i)}\right\},\left\{M^{(i)}\right\}\right.$, and $\hat{V}\left(\hat{Q}^{(1)}, \ldots, \hat{Q}^{(N)}\right)$ are the corresponding coordinates, momenta, masses, and potential energy, respectively], and

$$
\hat{H}_{b s}=\alpha \hat{q} \hat{F}\left(\hat{Q}^{(1)}, \ldots, \hat{Q}^{(N)}\right)
$$

is the system-bath coupling term, where $\alpha$ is the systembath coupling parameter. The system-bath coupling term, $\hat{H}_{b s}$, is assumed to be linearized in the vibrational coordinate, $\hat{q}$, which implies that VER takes place via the emission of one vibrational quantum. The force on the vibrational mode, $\hat{F}\left(\hat{Q}^{(1)}, \ldots, \hat{Q}^{(N)}\right)$, may be, and often is, a highly nonlinear function of the bath coordinates. The highly nonlinear nature of the force and the large frequency mismatch between the vibrational mode and the majority of the accepting modes, implies that the bath absorbs the energy via a multiphononlike process.

Given the general Hamiltonian above, the perturbative approach is based on the following three assumptions: (1) weak system-bath coupling, to the extent that Fermi's golden rule applies; (2) separation of time scales, such that the VER lifetime is much longer than the correlation time of the bathinduced force; (3) the rotating wave approximation (RWA), which amounts to the removal of rapidly oscillating terms and decoupling of population relaxation from phase relaxation. Under these conditions, the Bloch Redfield theory (BRT) leads to the following Master equation for the vibrational populations: $:^{51,58-67}$

$$
\begin{aligned}
\frac{d}{d t} P_{n}= & k_{n \leftarrow n+1} P_{n+1}+k_{n \leftarrow n-1} P_{n-1} \\
& -\left(k_{n+1 \leftarrow n}+k_{n-1 \leftarrow n}\right) P_{n},
\end{aligned}
$$

where

$$
k_{n \leftarrow n+1}=e^{\beta \hbar \omega} k_{n+1 \leftarrow n}=\alpha^{2} \frac{n+1}{\beta \hbar \omega} \frac{\beta}{2 \mu} \widetilde{C}(\omega) .
$$

Here, $\beta=\left(k_{B} T\right)^{-1}$, and

$$
\widetilde{C}(\omega)=\int_{-\infty}^{\infty} d \tau e^{i \omega \tau} C(\tau)
$$

is the Fourier transform (FT) of the free bath force-force correlation function (FFCF),

$$
C(\tau)=\left\langle\delta \hat{F}_{0}(\tau) \delta \hat{F}\right\rangle_{0},
$$

where $\langle\hat{A}\rangle_{0}=\operatorname{Tr}\left[e^{-\beta \hat{H}_{b}} \hat{A}\right] / \operatorname{Tr}\left[e^{-\beta \hat{H}_{b}}\right], \delta \hat{F}=\hat{F}-\langle\hat{F}\rangle_{0}$, and

$$
\delta \hat{F}_{0}(t)=e^{i \hat{H}_{b} t / \hbar} \delta \hat{F} e^{-i \hat{H}_{b} t / \hbar} .
$$

Thus, Eq. (6) puts the population relaxation rate constant in terms of the FT, at the vibrational frequency, of the quantummechanical FFCF, which is evaluated with the vibrational mode frozen at its equilibrium position $(\hat{q}=0)$.

As is well known, the population dynamics in Eq. (5) leads to an exponential decay of the vibrational energy, ${ }^{53,65,68}$

$$
\frac{d}{d t}\left\langle\delta \hat{H}_{s}\right\rangle=\sum_{n=0}^{\infty}(n+1 / 2) \hbar \omega \frac{d}{d t} P_{n}=-\frac{1}{T_{1}}\left\langle\delta \hat{H}_{s}\right\rangle,
$$

where $\delta \hat{H}_{s}=\hat{H}_{s}-\left\langle\hat{H}_{s}\right\rangle_{0}$, and $\left\langle\hat{H}_{s}\right\rangle_{0}=\hbar \omega / 2+\hbar \omega /\left(e^{\beta \hbar \omega}-1\right)$ is the vibrational energy at thermal equilibrium. The central quantity in Eq. (10) is the VER rate constant, $1 / T_{1}$, which is given by the Landau-Teller (LT) formula, ${ }^{10,45}$

$$
\frac{1}{T_{1}}=\frac{1-e^{-\beta \hbar \omega}}{\beta \hbar \omega} \frac{\beta}{2 \mu} \widetilde{C}(\omega) .
$$

The popularity of the LT formula ${ }^{1-3,38,51,53,55-57,63,65,69-82}$ should probably be attributed to the fact that it significantly cuts on the computational effort in comparison to the direct method. It does so in three major ways:

(1) The lifetime of the FFCF ( $\sim$ subpicoseconds) is typically much shorter than the VER lifetime (>picosecond). Thus, simulating the dynamics for a very short time can provide information on the rate of the much slower VER.

(2) The perturbative method requires an equilibrium MD simulation, which allows for more efficient sampling in comparison to the nonequilibrium simulation required by the direct method.

(3) The time step in a direct simulation of VER is dictated by the vibrational frequency, which usually corresponds to the fastest time scale. The FFCF is evaluated with the vibrational mode frozen $(\hat{q}=0)$, thereby making it possible to use a much larger time step.

Nevertheless, a consistent calculation of the VER rate constant via the LT formula also give rise to two major difficulties:

(a) Extracting the very small high-frequency Fourier components of the FFCF can become extremely difficult due to statistical noise accompanying all real-life simulations. ${ }^{1}$

(b) A numerically exact calculation of the quantum FFCF, or for that matter of any other many-body quantum correlation function, is beyond the reach of current state-of-the-art numerically-exact quantum dynamics simulations. $^{83}$ 
One approach for overcoming the numerical problem of calculating the high-frequency FT of the computed FFCF, is based on the extrapolation of the exponential gap law, which usually emerges at low frequencies, to much higher frequencies. ${ }^{84,85}$ Another, essentially equivalent, approach is to combine a short time expansion of the FFCF with a parameterized ansatz that exhibits an exponential gap law behavior at high frequencies, and whose FT can be calculated analytically. ${ }^{65,76,77,81,86-90}$ An alternative approach for overcoming the numerical noise is based on signal processing techniques, designed to isolate the desired FT (the signal) from the noise. ${ }^{63,91-93}$ However, even with the most powerful signal processing techniques, one is still unable to evaluate the FT at the required frequency for such systems as $\mathrm{O}_{2}$ in liquid $\mathrm{O}_{2} \cdot{ }^{76}$ Yet another recent attempt to deal with this problem was based on the hypothesis that high-frequency VER is dominated by the few instantaneous nearest neighbor solvent atoms. ${ }^{74}$ This approach has been demonstrated with relative success in the case of a homonuclear diatomic solute in an atomic Lennard-Jones (LJ) liquid. However, this method presently suffers from the following two shortcomings: (1) It fails for vibrational frequencies below a certain critical frequency, which may be difficult to estimate in general; (2) It is not clear how useful this approach would be in cases involving polar interactions and polyatomic solute and solvent.

The second difficulty involved in using the LT formula for calculating the VER rate constant has to do with the fact that one has to deal with the quantum FFCF, rather than the classical FFCF. The most popular approach for dealing with this difficulty is to first evaluate the classical VER rate constant, $1 / T_{1}^{\mathrm{Cl}}$, and then multiply the result by a frequency-dependent quantum correction factor, $A_{Q}(\omega),{ }^{1,53,54,56,57,75,79,92-101}$

$$
\frac{1}{T_{1}}=A_{Q}(\omega) \frac{1}{T_{1}^{\mathrm{Cl}}} .
$$

The classical VER rate constant is given by

$$
\frac{1}{T_{1}^{\mathrm{Cl}}}=\frac{\beta}{2 \mu} \widetilde{C}^{\mathrm{Cl}}(\omega)
$$

where $\quad \widetilde{C}^{\mathrm{Cl}}(\omega)=\int_{-\infty}^{\infty} d \tau e^{i \omega \tau} C^{\mathrm{Cl}}(\tau) \quad$ and $\quad C^{\mathrm{Cl}}(t)$ $=\left\langle\delta F_{0}(t) \delta F\right\rangle_{0}^{\mathrm{Cl}}$ is the classical $\mathrm{FFCF}\left(\langle\cdots\rangle_{0}^{\mathrm{Cl}}\right.$ corresponds to averaging over the classical Boltzmann phase space distribution and the time evolution of $\delta F_{0}(t)$ is governed by classical mechanics). The important point is that $C^{\mathrm{Cl}}(t)$ can be evaluated with relative ease from classical MD simulations. It should be noted that Eq. (12) amounts to nothing more than a reformulation of the original problem, since knowledge of the exact quantum correction factor, $A_{Q}(\omega)$, is equivalent to, and as difficult to obtain as, the exact quantum FFCF. However, educated guesses of $A_{Q}(\omega)$ can be introduced, which are based on the known general properties of quantum correlation functions (e.g., all quantum corrections must satisfy detailed balance) and/or the knowledge of what $A_{Q}(\omega)$ looks like in the very few cases where it is known exactly. However, a unique and general quantum correction factor is not known, and the estimates provided by the vari- ous quantum correction factors can differ by orders of magnitude, in particular when high-frequency vibrations are involved. ${ }^{54,56,79}$ For example, Egorov et al. have recently estimated $1 / T_{1}$ for $\mathrm{O}_{2}$ in liquid $\mathrm{O}_{2}$, at $70 \mathrm{~K}$, and found the following spread of values that were based on different quantum correction factors: $0.00095 \mathrm{~s}^{-1}$ (standard), $0.015 \mathrm{~s}^{-1}$ (harmonic), $270 \mathrm{~s}^{-1}$ (Egelstaff), $4030 \mathrm{~s}^{-1}$ (Schofield) (The experimental value under these conditions is $1 / T_{1}$ $=360 \mathrm{~s}^{-1}$ ). Similar disparity has also been observed in other systems. ${ }^{54,93}$ It should be noted that the Egelstaff and a mixed harmonic-Schofield quantum correction factors have been found to produce rather accurate results at high frequencies in this and other model systems. ${ }^{54,55,94}$ However, the ad hoc nature of this approach makes it difficult to assess the reasons for its success, or to predict how well it will perform in other systems. It should also be noted that several alternative approaches for calculating the quantummechanical FFCF have been proposed recently, which are based on centroid dynamics ${ }^{80-82,102}$ and imaginary-time path integral simulations. ${ }^{90}$ These promising approaches have been applied quite successfully to several model systems, although their general applicability and feasibility is not yet known.

The above mentioned difficulties involved in using the LT approach have motivated us to explore other approaches for the calculation of the VER rate constant. In this paper, we present such an alternative approach, which is based on linear response theory (LRT). The plan of this paper is as follows: The expression for the VER rate constant within the new approach is derived in Sec. II. Its relationship with the LT formula is established in Sec. III. In Sec. IV we demonstrate the application of the new method to a simple model system involving bilinear coupling to a harmonic bath. We conclude with a general discussion of the pros and cons of the LRT and LT approaches to VER in Sec. V.

\section{VIBRATIONAL ENERGY RELAXATION RATE CONSTANTS FROM LINEAR RESPONSE THEORY}

The new approach to VER proposed below is inspired by the reactive-flux method for calculating solution-phase reaction rate constants, ${ }^{103-107}$ and was motivated by the following similarities between VER and barrier crossing:

(a) Both VER and barrier crossing often follow rate kinetics which can be characterized by a single rate constant.

(b) Barrier crossing often occurs on a very slow time scale, due to the underlying rare event statistics. Hence, a direct approach, which would be based on nonequilibrium MD simulations, is usually impractical even if classical mechanics is used. The reactive-flux method circumvents this problem by expressing the reaction rate constant in terms of the flux-flux or the fluxHeaviside correlation functions, ${ }^{103-107}$ whose lifetimes are much shorter in comparison to the reaction rate. This situation is reminiscent of the perturbative approach to the calculation of VER rate constants, where the lifetime of the FFCF is much shorter than that of VER. 
(c) Similarly to VER of high-frequency vibrations, it is often important to account for quantum effects, such as tunneling and the zero point energy, when calculating the rates of reactions that involve light particles, such as proton transfer. In both cases, the quantum effects can be expressed in terms of a quantum mechanical correlation function.

A careful examination reveals, however, that despite these similarities, the LT approach to VER and the reactiveflux method are based on two different sets of assumptions: The LT formula originates from BRT (or equivalently from Fermi's golden rule), whereas the reactive-flux method is based on LRT. ${ }^{103,104}$ The BRT is based on the assumption of weak system-bath coupling, which should be clearly distinguished from the assumption of proximity to equilibrium, regardless of the strength of the system-bath coupling, which underlies LRT. Stimulated by the success of the reactive flux method, and noting the above mentioned similarities between VER and barrier crossing processes, we therefore set out to develop a new reactive-flux-like approach to VER.

Following the standard procedure of LRT, we consider an initial state which correspond to thermal equilibrium with respect to the perturbed Hamiltonian $\hat{H}+f \hat{A}$,

$$
\hat{\rho}(0)=\frac{e^{-\beta(\hat{H}+f \hat{A})}}{\operatorname{Tr}\left[e^{-\beta(\hat{H}+f \hat{A})}\right]} .
$$

Here, $\hat{H}$ is the actual Hamiltonian of the overall system [cf. Eq. (1)], $f$ is a coupling parameter, and $\hat{A}$ is the perturbation, which will be kept unspecified for the time being. We next assume that the perturbation, $f \hat{A}$, is small enough, such that $\hat{\rho}(0)$ can be substituted by its expansion to first order in $f$,

$$
\hat{\rho}(0) \approx \frac{e^{-\beta \hat{H}}}{Z}\left[1-\int_{0}^{\beta} d \lambda \delta \hat{A}(-i \hbar \lambda)\right],
$$

where $Z=\operatorname{Tr}\left(e^{-\beta \hat{H}}\right), \quad \delta \hat{A}=\hat{A}-\langle\hat{A}\rangle_{\mathrm{eq}}, \quad\langle\hat{A}\rangle_{\mathrm{eq}}=\operatorname{Tr}\left(e^{-\beta \hat{H}} \hat{A}\right) /$ $Z$, and $\hat{A}(-i \hbar \lambda)=e^{\lambda H} \hat{A} e^{-\lambda H}$. A measurement of VER is initiated by turning the perturbation off at $t=0$, and monitoring the expectation value of the vibrational energy as a function of time, as it progresses toward its equilibrium value. Substituting $\hat{\rho}(0)$ from Eq. (15) then yields

$$
\begin{aligned}
\left\langle\delta \hat{H}_{s}\right\rangle(t) & \equiv \operatorname{Tr}\left[\hat{\rho}(0) \delta \hat{H}_{s}(t)\right] \\
& =-f \int_{0}^{\beta} d \lambda\left\langle\delta \hat{A}(-i \hbar \lambda) \delta \hat{H}_{s}(t)\right\rangle_{\mathrm{eq}},
\end{aligned}
$$

where $\hat{H}_{s}(t)=e^{i \hat{H} t / \hbar} \hat{H}_{s} e^{-i \hat{H} t / \hbar}$.

Our basic hypothesis is that VER follows rate kinetics, and can therefore be characterized by a rate constant, $k$,

$$
\frac{\left\langle\delta \hat{H}_{s}\right\rangle(t)}{\left\langle\delta \hat{H}_{s}\right\rangle(0)}=e^{-k t} .
$$

The VER rate constant, $k$, can then be written in the following way:

$$
-\frac{\left\langle\delta \hat{H}_{s}\right\rangle(t)}{\left\langle\delta \hat{H}_{s}\right\rangle(0)}=k e^{-k t}=k \quad \text { at } t \ll k^{-1} .
$$

Substituting Eq. (16) into Eq. (18), and using the explicit expression for the vibrational energy flux,

$$
\hat{\hat{H}}_{s}=\frac{i}{\hbar}\left[\hat{H}, \hat{H}_{s}\right]=-\frac{\alpha}{\mu} \hat{p} \hat{F},
$$

then leads to the following general expression for the VER rate constant:

$$
k=\frac{\alpha}{\mu} \frac{\int_{0}^{\beta} d \lambda\langle\delta \hat{A}(-i \hbar \lambda)[\hat{p} \hat{F}](t)\rangle_{\mathrm{eq}}}{\int_{0}^{\beta} d \lambda\left\langle\delta \hat{A}(-i \hbar \lambda) \delta \hat{H}_{s}\right\rangle_{\mathrm{eq}}} .
$$

To the best of our knowledge, Eq. (20) is a new expression for the VER rate constant, and as such represents the main result of this paper. In analogy to the reactive-flux formalism, one expects the correlation function in Eq. (20) to be explicitly time-dependent during an initial short time period, following which it will reach a "plateau" (except for rapid oscillations that can be averaged out via the RWA). Reaching the plateau signals the onset of rate kinetics, and the VER rate constant will be given by the corresponding value of the correlation function. From BRT we know that the length of this transient time period is set by the lifetime of the FFCF. ${ }^{108}$ Hence, one would have to run the simulation for roughly the same length of time as would be required for calculating the FFCF.

\section{THE LANDAU-TELLER LIMIT}

The VER rate constant in Eq. (20), $k$, should be clearly distinguished from that given by the LT formula, $1 / T_{1}$ in Eq. (11). In this section we provide a detailed analysis of the conditions under which Eq. (20) reduces into the LT formula.

The LT formula is based on the three major assumptions underlying the BRT:

(1) Weak system-bath coupling, to the extent that Fermi's golden rule applies.

(2) Separation of time scales, such that the VER lifetime is much longer than the lifetime of the FFCF. The reduced dynamics becomes effectively Markovian under these conditions, and as a result the relaxation follows rate kinetics.

(3) The RWA, which amounts to the the averaging out of rapidly oscillating terms of frequency $2 \omega$, and decoupling of population relaxation from phase relaxation.

Thus, our goal would be to explicitly show that the LT formula emerges when these assumptions are imposed on Eq. (20).

In order to proceed, one first has to expand $k$ in Eq. (20) to second order in the system-bath coupling parameter, $\alpha$ [cf. Eq. (4)]. For simplicity, we chose to concentrate on a restricted class of relatively simple perturbations which have the following form:

$$
\hat{A}=\left(\hat{q}-\langle\hat{q}\rangle_{\mathrm{eq}}\right)^{n} \equiv(\delta \hat{q})^{n}, \quad n=1,2,3, \ldots .
$$


The first interesting observation is that the LT formula only emerges from Eq. (20) when $n$ is even. The reason for this can be traced back to the fact that, for an odd $n$, the $\alpha^{2}$ term vanishes in the expansion of $\left\langle\hat{H}_{s}\right\rangle(t)=-f(\alpha / \mu) \int_{0}^{\beta} d \lambda$ $\times\langle\delta \hat{A}(-i \hbar \lambda)[\hat{p} \hat{F}](t)\rangle_{\text {eq }}$. Thus, in the case of an odd $n$, VER occurs via a non-LT (non-BRT) pathway, induced by higher order terms in the perturbation expansion, which are associated with multiple-time force correlation functions (cf. Appendix A for more details). This observation demonstrates how one can take advantage of the flexibility with regard to choosing the perturbation in Eq. (20), in order to selectively explore different VER pathways.

For the choice of an even value of $n$, the $\alpha^{2}$ term is the lowest nonvanishing term in the expansion of $k$,

$$
k=\alpha^{2} k^{(2)}+o\left(\alpha^{4}\right) .
$$

$\alpha^{2} k^{(2)}$ can then be shown to reduce into $1 / T_{1}$ of the LT formula once we impose the additional two assumptions of BRT (separation of time scales and the RWA). We will now demonstrate this for $n=2$, i.e., for a perturbation of the form $\hat{A}=\left(q-\langle q\rangle_{\mathrm{eq}}\right)^{2}=(\delta \hat{q})^{2}$. The expansion of $k$ to second order in $\alpha$ is a rather lengthy exercise. An outline of the derivation is provided in Appendix A, and only the final result is given below,

$$
\alpha^{2} k^{(2)}(t)=\alpha^{2}[\mathcal{L}(t)+\mathcal{R}(t)+\mathcal{T}(t)]
$$

where,

$$
\begin{aligned}
\mathcal{L}(t)= & \frac{1}{\mu \hbar \omega} \operatorname{Re}\left\{\int_{0}^{t} d \tau e^{i \omega \tau} C(\tau)-\int_{0}^{t} d \tau e^{-i \omega \tau} C(\tau)\right\}, \\
\mathcal{R}(t)= & \frac{1}{\mu \hbar \omega} \frac{1}{2 \beta \hbar \omega}\left(e^{\beta \hbar \omega}-1\right)\left(1-e^{-\beta \hbar \omega}\right) \\
& \times \operatorname{Re}\left\{e^{2 i \omega t} \int_{0}^{t} d \tau e^{-i \omega \tau} C(\tau)\right. \\
& \left.-e^{-2 i \omega t} \int_{0}^{t} d \tau e^{i \omega \tau} C(\tau)\right\},
\end{aligned}
$$

and

$$
\begin{aligned}
\mathcal{T}(t)= & \frac{1}{\mu \omega}\left[\frac{1}{\beta \hbar \omega}\left(1-e^{-\beta \hbar \omega}\right) \sin (\omega t)\right. \\
& \times \operatorname{Re} \int_{0}^{\beta} d \lambda C(-t-i \hbar \lambda) e^{\lambda \hbar \omega} \\
& -\operatorname{Im}\left\{e^{-i \omega t} \int_{0}^{\beta} d \lambda C(-t-i \hbar \lambda) e^{\lambda \hbar \omega}\right. \\
& -\left(1-e^{-\beta \hbar \omega}\right) e^{-i \omega t} \frac{1}{\beta} \\
& \left.\left.\times \int_{0}^{\beta} d \lambda C(-t-i \hbar \lambda) \lambda e^{\lambda \hbar \omega}\right\}\right] .
\end{aligned}
$$

The LT formula emerges from Eq. (23) when the two additional approximations of BRT are imposed on it:
(1) Within BRT, rate kinetics is obtained at the Markovian limit, which is reached when $t$ becomes larger than the lifetime of the FFCF, denoted by $\tau_{c} . \tau_{c}$ is in fact the correlation time of the relevant bath fluctuations, which is very short in liquids $(\sim \mathrm{ps})$, particularly in comparison to the VER lifetime, $T_{1}$. In the case of Eq. (20), $\tau_{c}$ corresponds to the plateau time that signals the onset of rate kinetics. Assuming that $t>\tau_{c}$ in Eq. (23) then has two implications: (1) The transient term, $\mathcal{T}(t)$, vanishes $\left[C(-t-i \hbar \lambda) \rightarrow 0\right.$ at $\left.t>\tau_{c}\right]$; (2) the limit of the time integrals in the terms $\mathcal{L}(t)$ and $\mathcal{R}(t)$ can be extended to $\infty$.

(2) Decoupling the population and phase relaxations within BRT also requires the averaging out of terms which rotate rapidly with the frequency $2 \omega$ (the RWA). Applying this approximation to Eq. (23) leads to the removal of the rotating term $\mathcal{R}(t)$.

Thus, under these two approximations, $\alpha^{2} k^{(2)}$ reduces into $\alpha^{2} \lim _{t \rightarrow \infty} \mathcal{L}(t)$, which correspond to the VER rate constant. It is then easy to show, with the help of the following identities:

$$
\begin{aligned}
& \int_{0}^{\infty} d \tau e^{i \omega \tau} C(\tau)=\frac{1}{2} \widetilde{C}(\omega)+\frac{1}{2 \pi i} \mathcal{P} \int_{-\infty}^{\infty} d \omega^{\prime} \frac{\widetilde{C}\left(\omega^{\prime}\right)}{\omega^{\prime}-\omega}, \\
& \widetilde{C}(-\omega)=e^{-\beta \hbar \omega} \widetilde{C}(\omega),
\end{aligned}
$$

that

$$
\alpha^{2} k^{(2)} \approx \alpha^{2} \lim _{t \rightarrow \infty} \mathcal{L}(t)=\frac{1-e^{-\beta \hbar \omega}}{\beta \hbar \omega} \frac{\beta}{2 \mu} \widetilde{C}(\omega),
$$

which is identical to $1 / T_{1}$ in the LT formula, Eq. (11).

\section{APPLICATION IN THE CASE OF BILINEAR COUPLING TO A HARMONIC BATH}

In this section, we demonstrate the application of Eq. (20) to a simple model system involving bilinear coupling to a harmonic bath, for which Eqs. (11) and (20) can be calculated analytically. The facts that the classical and quantummechanical LT-based VER rate constants are identical, ${ }^{53}$ and that non-LT pathways do not contribute in this case, offer other attractive simplifications. It should be noted, however, that Eq. (20) is by no means limited to harmonic baths and/or bilinear coupling.

The bath is assumed to consist of independent harmonic modes,

$$
\hat{H}_{b}=\sum_{i=1}^{N}\left[\frac{\left(\hat{P}^{(i)}\right)^{2}}{2 M^{(i)}}+\frac{1}{2} M^{(i)}\left(\omega^{(i)}\right)^{2}\left(\hat{Q}^{(i)}\right)^{2}\right],
$$

and the force is assumed linear in the bath coordinates,

$$
\hat{F}=-\sum_{i=1}^{N} c^{(i)} \hat{Q}^{(i)} \text {. }
$$

For this model, the quantum mechanical and classical VER rate constants, as obtained from the LT formula, Eq. (11), are identical and given by ${ }^{53,55}$ 


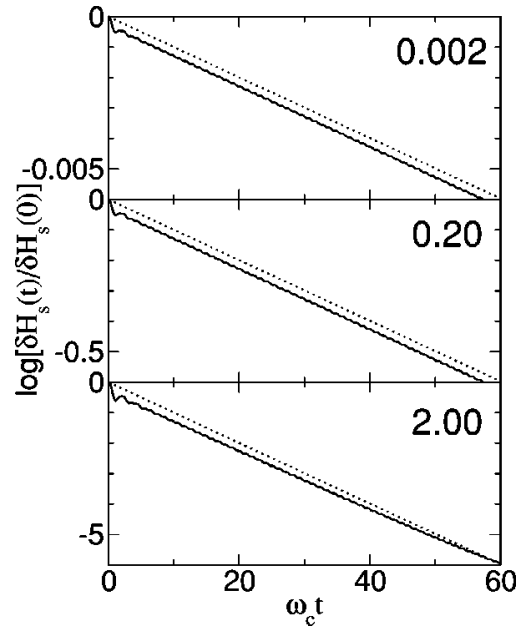

FIG. 1. Classical nonequilibrium VER profiles for a harmonic oscillator bilinearly coupled to a harmonic bath (solid lines). The plots are in a semi$\log$ format, such that linearity corresponds to rate kinetics. The calculations were performed for $\omega / \omega_{c}=3$ and $\beta \hbar \omega_{c}=4$. The three panels correspond to the indicated different values of the friction, $\eta / \mu \omega_{c}=0.002,0.20,2.00$. Also shown are the VER profiles predicted by the LT formula (dotted lines).

$$
\frac{1}{T_{1}}=\frac{J(\omega)}{\mu \omega},
$$

where $J(\omega)$ is the bath spectral density,

$$
J(\omega)=\frac{\pi}{2} \sum_{i} \frac{\left(c^{(i)}\right)^{2}}{M^{(i)} \omega^{(i)}} \delta\left(\omega-\omega^{(i)}\right)
$$

The actual calculations reported below were based on classical mechanics. Analytical treatment was made possible by transforming to the normal mode representation of the overall Hamiltonian, $\hat{H}$. An Ohmic spectral density with an exponential cutoff was used,

$$
J(\omega)=\eta \omega e^{-\omega / \omega_{c}},
$$

where $\omega_{c}$ is the bath cutoff frequency and $\eta$ is the friction coefficient (at the limit $\omega_{c} \rightarrow \infty$ ). All the calculations were performed for $\omega / \omega_{c}=3$ and $\beta \hbar \omega_{c}=4$.

Figure 1 shows the relaxation of the vibrational energy, on a semilog plot, as obtained from a calculation of the nonequilibrium dynamics, with the initial perturbation ( $q$ $\left.-\langle q\rangle_{\mathrm{eq}}\right)^{2}$ (solid lines). Also shown in Fig. 1 are the predictions based on the LT formula (dotted lines). The results are exhibited for three values of the friction, $\eta / \mu \omega_{c}$ $=0.002,0.2,2.0$. The classical equilibrium values of $\left\langle H_{s}\right\rangle$ are given by $\beta\left\langle H_{s}\right\rangle_{\mathrm{eq}}=1.0001,1.0072$, and 1.0824 for $\eta / \mu \omega_{c}$ $=0.002,0.2,2.0$, respectively. It should be noted that BRT predicts $\beta\left\langle H_{s}\right\rangle_{\mathrm{eq}}=\beta\left\langle H_{s}\right\rangle_{0}=1$, which corresponds to the zeroth order term in the expansion of $\left\langle H_{s}\right\rangle_{\mathrm{eq}}$ in powers of $\alpha$. This prediction is consistent at the lowest value of the friction considered, $\eta / \mu \omega_{c}=0.002$. However, significant deviations occur at the higher frictions, $\eta / \mu \omega_{c}=0.2,2.0$. These deviations are indicative of the breakdown of the weak system-bath coupling assumption, and departure from the region of parameter space where the LT formula is valid. The oscillations exhibited by the solid lines in Fig. 1, as opposed to the smooth exponential relaxation predicted by the LT

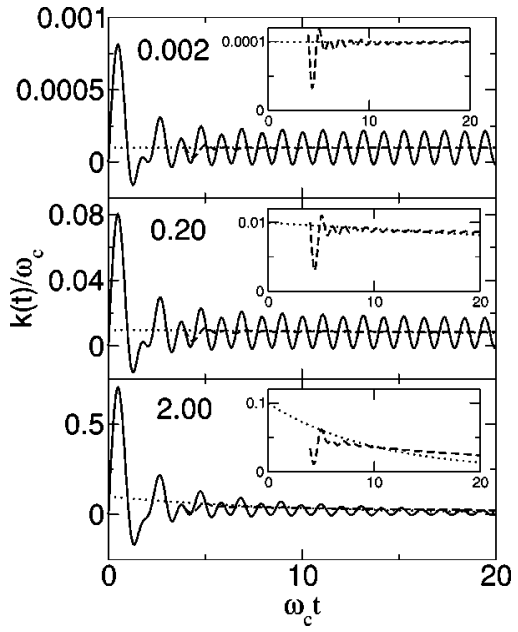

FIG. 2. The calculation of the (classical) VER rate constant via Eq. (20), for a harmonic oscillator bilinearly coupled to a harmonic bath (solid lines), for the indicated different values of the friction, $\eta / \mu \omega_{c}=0.002,0.20,2.00$. The dashed lines correspond to cumulative time averages over the solid lines (after reaching the plateau), whose goal is to recover the RWA and eliminate the oscillatory terms. Also shown are the predictions based on the LT formula, $\left(1 / T_{1}\right) e^{-t / T_{1}}$ (dotted line, see text for further discussion). The plateau region is shown in greater detail in the inserts. The calculations were performed for $\omega / \omega_{c}=3$ and $\beta \hbar \omega_{c}=4$.

formula, can be explained by the application of the RWA in the latter. If these rather small oscillations are averaged out, a seemingly exponential VER is found in all values of the friction considered, as indicated by the apparent linearity of the solid lines in Fig. 1 (in fact, a closer inspection reveals slight deviations from linearity at $\eta / \mu \omega_{c}=2.0$, which could probably not be experimentally resolved). The VER rate constant, which correspond to the slopes of the solid lines in Fig. 1 , is captured rather well by the LT formula at small and intermediate values of the friction $\left(\eta / \mu \omega_{c}=0.002,0.2\right)$. These cases demonstrate the consistency between the predictions of Eq. (20) and the LT formula under conditions where the latter is valid. However, a significant deviation between the nonequilibrium VER and the prediction of the LT formula appears at the higher friction $\left(\eta / \mu \omega_{c}=2.0\right)$. Thus, the case of $\eta / \mu \omega_{c}=2.0$ provides an example for VER that follows rate kinetics under conditions of strong system-bath coupling, where the LT formula is not valid.

In Fig. 2, we show the results obtained by using Eq. (20) in order to calculate the VER rate constant, for the same three values of the friction as in Fig. 1. It should be noted that Eq. (20) is directly related to the time-derivative of the nonequilibrium curves in Fig. 1 (as long as $k t \ll 1$ ). As expected, $k$ exhibits an initial transient time dependence, which is perturbation-dependent, before reaching a "plateau," which signals the onset of rate kinetics (solid lines). It should be noted that $k$ oscillates at a frequency of $2 \omega$ at the plateau, which can be explained by the absence of the RWA. These oscillations can be averaged out rather effectively, resulting with the sought after VER rate constant (dashed lines). The latter coincides with the prediction of the LT formula (dotted lines) at the lowest value of the friction, $\eta / \mu \omega_{c}=0.002$. However, significant deviations appear as the friction increases. It should be noted that Eq. (20) is based on the 
assumption that $k t \ll 1$, such that $k e^{-k t} \approx k$ [cf. Eq. (18)]. This means that the plateau value should decrease slowly like $e^{-k t}$. When comparing to the LT formula, we therefore use $\left(1 / T_{1}\right) e^{-t / T_{1}}$. One can clearly see that the LT formula overestimates the VER rate constant as the friction increases, which is consistent with the nonequilibrium relaxation exhibited in Fig. 1.

\section{DISCUSSION}

The above analysis links the two major theoretical frameworks for obtaining rate constants, namely BRT and LRT, in the case of VER. It shows that the LT formula, originally derived from BRT, can also be obtained from LRT under the following conditions: (1) an appropriate choice of the perturbed state; (2) taking the limit of weak system-bath coupling (second order in $\alpha$ ); (3) the RWA. This result ensures that the new LRT expression, Eq. (20), will give the same result as the LT formula, Eq. (11), as long as the underlying assumptions of BRT are valid. However, it should be emphasized that Eq. (20) is by no means equivalent to the LT formula, and that the former has several important advantages over the latter:

(a) Equation (20) is, in some sense, an intermediate between the direct method and the perturbative method. As for the perturbative method, the correlation function in Eq. (20) can be obtained by simulating the equilibrium dynamics for a short length of time, which is dictated by the lifetime of the FFCF, rather than that of the actual VER. At the same time, the simulation should be performed with a flexible vibrational mode, similarly to the direct method. Including a flexible vibrational mode implies a shorter time step, which parallels the increasing difficulty involved in obtaining the highfrequency tail of the FT of the FFCF within the LT approach. The question of which method is more computationally intensive in this respect will be addressed in a future paper.

(b) Unlike the perturbative approach, an approach based on Eq. (20) can tell us if rate kinetics is a valid assumption-failure to reach a well defined plateau indicates that the VER cannot be characterized by a rate constant. It should be noted that within the LT approach, rate kinetics is an outcome of the inter-related assumptions of weak system-bath coupling and Markovity. However, rate kinetics may arise from other sources. For example, rate kinetics may well be the outcome of rare event statistics, as in the case of barrier crossing, or may correspond to higher order terms in the system-bath coupling, as in the case of choosing an odd value of $n$ in Eq. (21). In such cases, the LT formula will not give the correct VER rate constant, but Eq. (20) will!

(c) Equation (20) actually corresponds to a family of expressions for the VER rate constant, which differ with respect to the choice of initial perturbation, $\hat{A}$. This flexibility translates into several advantages:

(1) Initial states that correspond to different choices of $\hat{A}$ can relax via different pathways, which may correspond to different VER rate constants. Thus, carefully choosing the initial perturbation can allow us to selectively focus on one VER pathway at a time.

(2) Modern methodologies for computing approximate quantum mechanical real-time correlation functions are often restricted to, or work better for, certain classes of correlation functions. One can therefore take advantage of the flexibility in choosing $\hat{A}$ in order to fine tune the expression for the VER rate constant, and put it in terms of a convenient correlation function. An example for such an application, in the case of the centroid molecular dynamics (CMD) method, will be discussed in a separate paper.

The flexibility allowed for by the new approach actually goes beyond the choice of $\hat{A}$. For example, we show in Appendix $B$ how the choice of a non-Kubo-transformed initial state of the form

$$
\hat{\rho}(0)=\frac{e^{-\beta \hat{H}}}{Z}+\frac{1}{2 Z}\left[\delta \hat{A} e^{-\beta \hat{H}}+e^{-\beta \hat{H}} \delta \hat{A}\right]
$$

can also lead to the LT formula in the appropriate limit.

To summarize, the goal of this paper was to introduce a new LRT-based method for calculating quantum mechanical VER rate constants, and establish its relationship to the standard LT approach. We have also discussed the advantages of the LRT approach over the LT approach, and concluded that the former is more general, flexible and informative than the latter. Both methods appear comparable with respect to the computational effort that they require. More specifically, they both require an equilibrium MD simulation over a period of time which is dictated by the lifetime of the FFCF. LRT requires that the simulation is performed with flexible vibrational modes, which would appear to enhance the computational effort in comparison to the LT approach, where the simulation is done with frozen vibrational modes. However, a higher frequency also translates into more computational work in the LT approach, due to the rapidly increasing difficulty of evaluating the high-frequency tail of the FT of the FFCF. We therefore expect the new method to be at least as computationally feasible as the LT method. Finally, taking into account the additional advantages of LRT seems to suggest that there may be cases where it could be the method of choice for treating high-frequency VER rate constants.

\section{ACKNOWLEDGMENT}

This project was partially supported by the National Science Foundation FOCUS Center, Grant No. 0114336.

\section{APPENDIX A: THE DERIVATION OF THE LANDAU-TELLER FORMULA FROM LINEAR RESPONSE THEORY}

In this section we outline the derivation of the LT formula, Eq. (11), from the LRT expression for the VER rate constant, Eq. (20). To this end, we choose the perturbation 


$$
\hat{A}=(\delta \hat{q})^{2}=\left(\hat{q}-\langle\hat{q}\rangle_{\mathrm{eq}}\right)^{2} .
$$

Substituting it into Eq. (20) then yields

$$
k=\frac{\frac{\alpha}{\mu} \int_{0}^{\beta} d \lambda \operatorname{Tr}\left\{e^{-\beta \hat{H}} \delta(\delta \hat{q})^{2}(-i \hbar \lambda)[\hat{p} \hat{F}](t)\right\}}{\int_{0}^{\beta} d \lambda \operatorname{Tr}\left\{e^{-\beta \hat{H}} \delta(\delta \hat{q})^{2}(-i \hbar \lambda) \delta \hat{H}_{s}\right\}} .
$$

In order to proceed, one has to find the leading terms when $k$ is expanded in powers of $\alpha$. It can be readily shown that the leading term in the expansion of the denominator is of zeroth order in $\alpha$, while that in the expansion of the numerator is second order in $\alpha$. Thus, the leading term in the expansion of $k$ is also of second order in $\alpha$, and the corresponding coefficient is given by

$$
\begin{aligned}
k^{(2)}= & \frac{1}{\beta \hbar^{2}} \frac{\left(e^{\beta \hbar \omega}-1\right)^{2}}{e^{\beta \hbar \omega}} \frac{1}{Z_{s}^{0} Z_{b}^{0}} \\
& \times\left[\int_{0}^{\beta} d \lambda \operatorname{Tr}\left\{e^{-\beta \hat{H}} \delta(\delta \hat{q})^{2}(-i \hbar \lambda)[\hat{p} \hat{F}](t)\right\}\right]_{1} .
\end{aligned}
$$

Here, $Z_{s}^{0}=\operatorname{Tr}_{s}\left(e^{-\beta \hat{H}_{s}}\right), Z_{b}^{0}=\operatorname{Tr}_{b}\left(e^{-\beta \hat{H}_{b}}\right)$, and $[\cdots]_{1}$ is the first order term, with respect to $\alpha$, of the corresponding expression. It should be noted that $e^{-\beta \hat{H}}, \delta(\delta \hat{q})^{2}(-i \hbar \lambda)$ and $[\hat{p} \hat{F}](t)$ all depend explicitly on $\alpha$, and have nonvanishing first order terms when expanded in powers of $\alpha$,

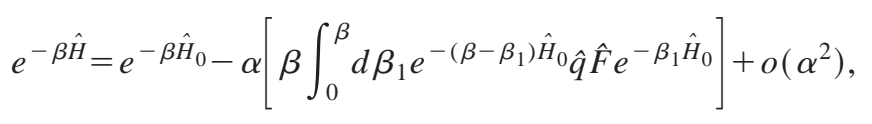

$$
\begin{aligned}
\delta(\delta \hat{q})^{2}(-i \hbar \lambda)= & \hat{q}_{0}^{2}(-i \hbar \lambda)-\left\langle\hat{q}^{2}\right\rangle_{0}+\alpha \frac{\hbar}{\mu \omega} \hat{q}_{0}(-i \hbar \lambda) \\
& \times\left[\int _ { 0 } ^ { \lambda } d \lambda _ { 1 } \left(e^{\left(\lambda-\lambda_{1}\right) \hbar \omega}\right.\right. \\
& \left.-e^{-\left(\lambda-\lambda_{1}\right) \hbar \omega}\right) \hat{F}_{0}\left(-i \hbar \lambda_{1}\right) \\
& \left.+\frac{2}{\mu \omega^{2}}\langle\hat{F}\rangle_{0} \hat{q}_{0}(-i \hbar \lambda)\right]+o\left(\alpha^{2}\right),
\end{aligned}
$$

$$
\begin{aligned}
{[\hat{p} \hat{F}](t)=} & \hat{p}_{0}(t) \hat{F}_{0}(t)+\alpha \frac{i}{\hbar} \int_{0}^{t} d t_{1}\left[\hat{q}_{0}\left(t_{1}\right) \hat{p}_{0}(t) \hat{F}_{0}\left(t_{1}\right) \hat{F}_{0}(t)\right. \\
& \left.-\hat{p}_{0}(t) \hat{q}_{0}\left(t_{1}\right) \hat{F}_{0}(t) \hat{F}_{0}\left(t_{1}\right)\right]+o\left(\alpha^{2}\right) .
\end{aligned}
$$

Here, $\langle\hat{A}\rangle_{0}=\operatorname{Tr}\left(\hat{A} e^{-\beta \hat{H}_{0}}\right) / Z^{0}$ and $\hat{A}_{0}(\tau)=e^{i \hat{H}_{0} \tau / \hbar} \hat{A} e^{-i \hat{H}_{0} \tau / \hbar}$.
Thus,

$$
\begin{aligned}
& {\left[\int_{0}^{\beta} d \lambda \operatorname{Tr}\left\{e^{-\beta \hat{H}} \delta(\delta \hat{q})^{2}(-i \hbar \lambda)[\hat{p} \hat{F}](t)\right\}\right]_{1}} \\
& \quad \int_{0}^{\beta} d \lambda \operatorname{Tr}\left\{\left[e^{-\beta \hat{H}}\right]_{1}\left(\hat{q}_{0}^{2}(-i \hbar \lambda)-\left\langle\hat{q}^{2}\right\rangle_{0}\right) \hat{p}_{0}(t) \hat{F}_{0}(t)\right\} \\
& \quad+\int_{0}^{\beta} d \lambda \operatorname{Tr}\left\{e^{-\beta \hat{H}_{0}}\left[\delta(\delta \hat{q})^{2}(-i \hbar \lambda)\right]_{1} \hat{p}_{0}(t) \hat{F}_{0}(t)\right\} \\
& \quad+\int_{0}^{\beta} d \lambda \operatorname{Tr}\left\{e^{-\beta \hat{H}_{0}}\left(\hat{q}_{0}^{2}(-i \hbar \lambda)-\left\langle\hat{q}^{2}\right\rangle_{0}\right)[\hat{p} \hat{F}]_{1}(t)\right\} .
\end{aligned}
$$

In order to proceed, one has to substitute the corresponding zeroth and first order terms from Eqs. (A4) into Eq. (A5). The resulting equation can then be further simplified to yield Eq. (23). More specifically, the first two terms on the R.H.S. of Eq. (A5) give rise to the transient term $\mathcal{T}(t)$ of Eq. (26), while the third term give rise to the rotating term, $\mathcal{R}(t)$ in Eq. (25), and the LT term, $\mathcal{L}(t)$ in Eq. (24).

We next consider what happens when one substitutes the perturbation $\hat{A}=\delta \hat{q} \equiv \hat{q}-\langle\hat{q}\rangle_{\text {eq }}$ into Eq. (20),

$$
k=\frac{\frac{\alpha}{\mu} \int_{0}^{\beta} d \lambda \operatorname{Tr}\left\{e^{-\beta \hat{H}} \delta \hat{q}(-i \hbar \lambda)[\hat{p} \hat{F}](t)\right\}}{\int_{0}^{\beta} d \lambda \operatorname{Tr}\left\{e^{-\beta \hat{H}} \delta \hat{q}(-i \hbar \lambda) \delta \hat{H}_{s}\right\}} .
$$

It can be readily shown that in this case the leading terms in the expansion of the denominator and numerator are of first order in $\alpha$. Thus, the leading term in the expansion of $k$ is of zeroth order, and is given by

$$
k^{(0)}=-\omega \sin (\omega t) .
$$

This term obviously cannot describe rate kinetics. The next term after the leading term in the expansion of $k$ is of second order in $\alpha$, and correspond to the ratio of the third order term in the expansion of the numerator and the first order term in the expansion of the denominator. Although this term may well lead to rate kinetics, it will not be LT-like. This is because the force enters into this term in the form of two-time force correlation functions, $\left\langle\hat{F}_{0}\left(\tau_{1}\right) \hat{F}_{0}\left(\tau_{2}\right) \hat{F}\right\rangle_{0}$, rather than the one-time force correlation function, $\left\langle\hat{F}_{0}(\tau) \hat{F}\right\rangle_{0}$, that appear in the LT formula.

This somewhat surprising result originates from the fact that the perturbation $\hat{A}=\delta \hat{q} \equiv \hat{q}-\langle\hat{q}\rangle_{\text {eq }}$ amounts to shifting the harmonic potential without changing its frequency [this should be contrasted to the quadratic perturbation in Eq. (A1) that does change the frequency]. Thus, the equilibrium energy of the oscillator at thermal equilibrium, to zero-order in the system-bath coupling, does not change when $\hat{A}=\delta \hat{q}$ $\equiv \hat{q}-\langle\hat{q}\rangle_{\text {eq }}$. As a result, VER becomes dominated by higher order terms. More specifically, one has to remember that the numerator in Eq. (20) corresponds to $\left\langle\hat{H}_{s}\right\rangle \equiv \operatorname{Tr}\left(\dot{\hat{\rho}}_{s} \hat{H}_{s}\right)$ [cf. Eq. (18)], and that the BRT can only account for terms which are up to second order in $\alpha$ in the expansion of $\dot{\hat{\rho}}_{s}$. The zero-order term in the expansion of $k$, Eq. (A7), comes 
from the first order term in the expansion of $\left\langle\dot{\hat{H}}_{s}\right\rangle$. Such terms correspond in the BRT to a bath-induced renormalization of the system Hamiltonian and as such cannot lead to relaxation dynamics. The second order term in the expansion of $k$ comes from the third order term in the expansion of $\left\langle\hat{H}_{s}\right\rangle$ which is not accounted for by the BRT. Such higherthan-second-order terms are obtained when one goes beyond second order perturbation theory, ${ }^{108}$ and represent non-LT VER pathways. Thus, the above analysis indicates that non-LT pathways can be accessed and studied by preparing the system in certain initial nonequilibrium states. It should also be noted that the second order term in the expansion of $\left\langle\hat{H}_{s}\right\rangle$ vanishes because of the asymmetry of the perturbation $\hat{A}=\delta \hat{q}$, and that non-LT VER will emerge for other asymmetrical forms of $\hat{A}$, such as $\hat{A}=(\delta \hat{q})^{n}$ with $n=3,5,7, \ldots$.

It is also interesting to note that the emergence of non-LT VER is intimately related to the fact that the system and bath are initially correlated and that the state of the bath is in fact affected by the system. Under the assumptions of the BRT, an uncorrelated initial state of the form $\hat{\rho}(0)$ $=\left(e^{-\beta \hat{H}_{b}} / Z_{b}^{0}\right) \otimes \hat{\rho}_{s}(0)$ will generally lead to LT VER, except for the trivial case where the vibrational mode is initially equilibrated, $\hat{\rho}_{s}(0)=e^{-\beta \hat{H}_{s}} / Z_{s}^{0}$. However, the initial state in LRT, Eq. (15), includes additional system-bath correlated terms which arise from the system-bath coupling term, Eq. (4). More specifically, $\hat{\rho}(0)$ in Eq. (15) can be written in the following way:

$$
\hat{\rho}(0)=\frac{e^{-\beta \hat{H}_{b}}}{Z_{b}^{0}} \otimes \hat{\rho}_{s}^{(0)}(0)+\sum_{n=1}^{\infty} \alpha^{n} \hat{\rho}^{(n)}(0),
$$

where

$$
\hat{\rho}_{s}^{(0)}(0)=\frac{e^{-\beta \hat{H}_{s}}}{Z_{s}^{0}}\left(1-f \int_{0}^{\beta} d \lambda \delta \hat{A}_{0}(-i \hbar \lambda)\right),
$$

is independent of $\alpha$, and $\hat{\rho}^{(n)}(0)$ represents the $n$th order system-bath correlated term, which intrinsically cannot be written in the form $\left(e^{-\beta \hat{H}_{b}} Z_{b}^{0}\right) \otimes \hat{\rho}_{s}(0)$. Thus, LT VER is bound to emerge when $\operatorname{Tr}\left(\hat{\rho}_{s}^{(0)}(0) \delta \hat{H}_{s}\right) \neq 0$, e.g., when $n$ is even in Eq. (21). However, situations where $\operatorname{Tr}\left(\hat{\rho}_{s}^{(0)}(0) \delta \hat{H}_{s}\right)=0$, e.g., in the case of an odd value of $n$ in Eq. (21), will be dominated by the higher order correlated terms and lead to non-LT VER.

\section{APPENDIX B: VIBRATIONAL ENERGY RELAXATION WITH A NON-KUBO-TRANSFORMED INITIAL STATE}

The initial state of the overall system can be written in terms of the following general form:

$$
\hat{\rho}(0)=\frac{e^{-\beta \hat{H}}}{Z}+\hat{\Delta},
$$

where the deviation from equilibrium, $\hat{\Delta}$, must satisfy $\operatorname{Tr}(\hat{\Delta})=0, \hat{\Delta}^{\dagger}=\hat{\Delta}$, and as long as $\hat{\rho}(0)$ is positive. Following a LRT-like procedure then yields the following expression for the relaxation of $\left\langle\hat{H}_{s}\right\rangle$ to equilibrium:

$$
\left\langle\delta \hat{H}_{s}\right\rangle(t)=\operatorname{Tr}\left[\hat{\Delta} \delta \hat{H}_{s}(t)\right] .
$$

It should be noted that Eq. (B2) applies to any initial state, regardless of its proximity to equilibrium, and is therefore more general than the standard LRT. LRT can obviously be retrieved from Eq. (B2) by substituting $\hat{\Delta}$ $=-f \int_{0}^{\beta} d \lambda e^{-(\beta-\lambda) \hat{H}} \delta \hat{A} e^{-\lambda \hat{H}} / Z$.

Provided that $\left\langle\delta \hat{H}_{s}\right\rangle(t)$ follows rate kinetics, one can then put the rate constant in the following form:

$$
k=-\frac{\operatorname{Tr}\left[\hat{\Delta} \hat{H}_{s}(t)\right]}{\operatorname{Tr}\left[\hat{\Delta} \delta \hat{H}_{s}\right]} .
$$

It should be noted that the actual value of the rate constant, and even the actual validity of rate kinetics, may depend on the choice of $\hat{\Delta}$. An example for this has been given above within the LRT, where $\hat{A}=\delta \hat{q}$ and $\hat{A}=(\delta \hat{q})^{2}$ have been shown to lead to different VER rate constants. At the same time, it is plausible that choices of the deviation from equilibrium other than that dictated by LRT can be used in order to obtain the same rate constant. Such alternatives may also be easier to handle and simulate than the Kubo-transformed form dictated by LRT.

We now demonstrate this idea for a deviation from equilibrium of the following form:

$$
\hat{\Delta}=-\frac{f}{2 Z}\left(\delta(\delta \hat{q})^{2} e^{-\beta \hat{H}}+e^{-\beta \hat{H}} \delta(\delta \hat{q})^{2}\right) .
$$

Substituting Eq. (B4) into Eq. (B3) yields

$$
k=\frac{\alpha}{\mu} \frac{\operatorname{Re}\left\langle[\hat{p} \hat{F}](t) \delta(\delta \hat{q})^{2}\right\rangle_{\mathrm{eq}}}{\operatorname{Re}\left\langle\delta(\delta \hat{q})^{2} \delta \hat{H}_{s}\right\rangle_{\mathrm{eq}}} .
$$

As for the LRT expression, the leading term in the expansion of $k$ is second order in $\alpha$, and is given by

$$
\alpha^{2} k^{(2)}(t)=\alpha^{2}\left[\mathcal{L}^{\prime}(t)+\mathcal{R}^{\prime}(t)+\mathcal{T}^{\prime}(t)\right],
$$

where

$$
\begin{aligned}
\mathcal{L}^{\prime}(t)= & \frac{1}{2 \mu \hbar \omega}\left(1-e^{-\beta \hbar \omega}\right) \operatorname{Re}\left\{\int_{0}^{t} d \tau e^{i \omega \tau} C(\tau)\right. \\
& \left.+e^{\beta \hbar \omega} \int_{0}^{t} d \tau e^{-i \omega \tau} C(\tau)\right\} \\
\mathcal{R}^{\prime}(t)= & -\frac{1}{2 \mu \hbar \omega}\left(e^{\beta \hbar \omega}-1\right) \\
& \times \operatorname{Re}\left\{e^{-\beta \hbar \omega} e^{2 i \omega t} \int_{0}^{t} d \tau e^{-i \omega \tau} C(\tau)\right. \\
& \left.+e^{-2 i \omega t} \int_{0}^{t} d \tau e^{i \omega \tau} C(\tau)\right\} \\
\mathcal{T}^{\prime}(t)= & \frac{\hbar}{e^{\beta \hbar \omega}-1} \sin (2 \omega t) \int_{0}^{\beta} d \lambda C(-i \hbar \lambda) \\
& \times\left[e^{(\beta-\lambda) \hbar \omega}+e^{-(\beta-\lambda) \hbar \omega}\right] .
\end{aligned}
$$

The terms $\mathcal{R}^{\prime}(t)$ and $\mathcal{T}^{\prime}(t)$ can then be averaged out via the RWA, while the term $\mathcal{L}^{\prime}(t)$ reduces into the LT formula at $t>\tau_{c}$. 
${ }^{1}$ D. W. Oxtoby, Adv. Chem. Phys. 47, 487 (1981).

${ }^{2}$ D. W. Oxtoby, Annu. Rev. Phys. Chem. 32, 77 (1981).

${ }^{3}$ D. W. Oxtoby, J. Phys. Chem. 87, 3028 (1983).

${ }^{4}$ J. Chesnoy and G. M. Gale, Ann. Phys. (Paris) 9, 893 (1984).

${ }^{5}$ J. Chesnoy and G. M. Gale, Adv. Chem. Phys. 70, 297 (1988).

${ }^{6}$ C. B. Harris, D. E. Smith, and D. J. Russell, Chem. Rev. 90, 481 (1990).

${ }^{7}$ D. W. Miller and S. A. Adelman, Int. J. Radiat. Phys. Chem. 13, 359 (1994).

${ }^{8}$ R. M. Stratt and M. Maroncelli, J. Phys. Chem. 100, 12981 (1996).

${ }^{9}$ T. Elsaesser and W. Kaiser, Annu. Rev. Phys. Chem. 42, 83 (1991).

${ }^{10}$ J. C. Owrutsky, D. Raftery, and R. M. Hochstrasser, Annu. Rev. Phys. Chem. 45, 519 (1994).

${ }^{11}$ W. F. Calaway and G. E. Ewing, J. Chem. Phys. 63, 2842 (1975).

${ }^{12}$ S. R. J. Brueck and R. M. Osgood, Chem. Phys. Lett. 39, 568 (1976).

${ }^{13}$ A. Laubereau and W. Kaiser, Rev. Mod. Phys. 50, 607 (1978).

${ }^{14}$ B. Faltermeier, R. Protz, M. Maier, and E. Werner, Chem. Phys. Lett. 74, 425 (1980).

${ }^{15}$ B. Faltermeier, R. Protz, and M. Maier, Chem. Phys. 62, 377 (1981).

${ }^{16}$ M. Chateau et al., J. Chem. Phys. 71, 4799 (1979).

${ }^{17}$ C. Delalande and G. M. Gale, J. Chem. Phys. 73, 1918 (1980).

${ }^{18}$ P. Roussignol, C. Delalande, and G. M. Gale, Chem. Phys. 70, 319 (1982).

${ }^{19}$ E. J. Heilweil, F. E. Doany, R. Moore, and R. M. Hochstrasser, J. Chem. Phys. 76, 5632 (1982).

${ }^{20}$ E. J. Heilweil, M. P. Casassa, R. R. Cavanagh, and J. C. Stephenson, Chem. Phys. Lett. 117, 185 (1985).

${ }^{21}$ E. J. Heilweil, M. P. Casassa, R. R. Cavanagh, and J. C. Stephenson, J. Chem. Phys. 85, 5004 (1986).

${ }^{22}$ A. L. Harris, J. K. Brown, and C. B. Harris, Annu. Rev. Phys. Chem. 39, 341 (1988).

${ }^{23}$ M. E. Paige, D. J. Russell, and C. B. Harris, J. Chem. Phys. 85, 3699 (1986).

${ }^{24}$ J. C. Owrutsky et al., Chem. Phys. Lett. 184, 368 (1991).

${ }^{25}$ A. Moustakas and E. Weitz, J. Chem. Phys. 98, 6947 (1993).

${ }^{26}$ D. A. V. Kliner, J. C. Alfano, and P. F. Barbara, J. Chem. Phys. 98, 5375 (1993).

${ }^{27}$ D. Zimdars et al., Phys. Rev. Lett. 70, 2718 (1993).

${ }^{28}$ N. Pugliano, A. Z. Szarka, S. Gnanakaran, and R. M. Hochstrasser, J. Chem. Phys. 103, 6498 (1995).

${ }^{29}$ M. E. Paige and C. B. Harris, Chem. Phys. 149, 37 (1990).

${ }^{30}$ A. Salloum and H. Dubost, Chem. Phys. 189, 179 (1994).

${ }^{31}$ A. Tokmakoff, B. Sauter, and M. D. Fayer, J. Chem. Phys. 100, 9035 (1994).

${ }^{32}$ A. Tokmakoff and M. D. Fayer, J. Chem. Phys. 103, 2810 (1995).

${ }^{33}$ R. S. Urdahl et al., J. Chem. Phys. 107, 3747 (1997).

${ }^{34}$ J. C. Owrutsky, M. Li, B. Locke, and R. M. Hochstrasser, J. Phys. Chem. 99, 4842 (1995).

${ }^{35}$ R. Laenen, C. Rauscher, and A. Laubereau, Phys. Rev. Lett. 80, 2622 (1998).

${ }^{36} \mathrm{~S}$. Woutersen, U. Emmerichs, H. Nienhuys, and H. J. Bakker, Phys. Rev. Lett. 81, 1106 (1998).

${ }^{37}$ D. J. Myers et al., J. Chem. Phys. 109, 5971 (1998).

${ }^{38}$ D. E. Sagnella et al., Proc. Natl. Acad. Sci. U.S.A. 96, 14324 (1999).

${ }^{39}$ P. Hamm, M. Lim, and R. M. Hochstrasser, J. Chem. Phys. 107, 10523 (1997).

${ }^{40}$ T. A. Litovitz, J. Chem. Phys. 26, 469 (1957).

${ }^{41}$ W. M. Madigosky and T. A. Litovitz, J. Chem. Phys. 34, 489 (1961).

${ }^{42}$ P. K. Davis and I. Oppenheim, J. Chem. Phys. 57, 505 (1972).

${ }^{43}$ D. W. Oxtoby, Mol. Phys. 34, 987 (1977).

${ }^{44}$ D. J. Nesbitt and J. T. Hynes, J. Chem. Phys. 77, 2130 (1982).

${ }^{45}$ R. Zwanzig, J. Chem. Phys. 34, 1931 (1961).

${ }^{46}$ P. S. Dardi and R. I. Cukier, J. Chem. Phys. 89, 4145 (1988).

${ }^{47}$ P. S. Dardi and R. I. Cukier, J. Chem. Phys. 95, 98 (1991).

${ }^{48}$ J. K. Brown, C. B. Harris, and J. C. Tully, J. Chem. Phys. 89, 6687 (1988).

${ }^{49}$ R. M. Whitnell, K. R. Wilson, and J. T. Hynes, J. Phys. Chem. 94, 8625 (1990).

${ }^{50}$ R. M. Whitnell, K. R. Wilson, and J. T. Hynes, J. Chem. Phys. 96, 5354 (1992).

${ }^{51}$ F. E. Figueirido and R. M. Levy, J. Chem. Phys. 97, 703 (1992).

${ }^{52}$ S. Jang, Y. Pak, and G. A. Voth, J. Phys. Chem. A 103, 10289 (1999).

${ }^{53}$ J. S. Bader and B. J. Berne, J. Chem. Phys. 100, 8359 (1994).

${ }^{54}$ S. A. Egorov and J. L. Skinner, Chem. Phys. Lett. 293, 439 (1998).
${ }^{55}$ S. A. Egorov and B. J. Berne, J. Chem. Phys. 107, 6050 (1997).

${ }^{56}$ S. A. Egorov, K. F. Everitt, and J. L. Skinner, J. Phys. Chem. A 103, 9494 (1999).

${ }^{57}$ B. J. Berne, J. Jortner, and R. Gordon, J. Chem. Phys. 47, 1600 (1967).

${ }^{58}$ A. G. Redfield, IBM J. Res. Dev. 1, 19 (1957).

${ }^{59}$ R. K. Wangsness and F. Bloch, Phys. Rev. 89, 728 (1953).

${ }^{60}$ C. P. Slichter, Principles of Magnetic Resonance (Springer-Verlag, Berlin, 1990).

${ }^{61}$ A. Abragam, The Principles of Nuclear Magnetism (Oxford, London, 1961).

${ }^{62}$ J. M. Jean, R. A. Friesner, and G. R. Fleming, J. Chem. Phys. 96, 5827 (1992).

${ }^{63}$ H. Gai and G. A. Voth, J. Chem. Phys. 99, 740 (1993).

${ }^{64}$ W. T. Pollard and R. A. Friesner, J. Chem. Phys. 100, 5054 (1994).

${ }^{65}$ S. A. Egorov and J. L. Skinner, J. Chem. Phys. 105, 7047 (1996).

${ }^{66}$ E. Geva, E. Rosenman, and D. J. Tannor, J. Chem. Phys. 113, 1380 (2000).

${ }^{67}$ N. G. V. Kampen, Stochastic Processes in Physics and Chemistry (Elsevier, Amsterdam, 1992).

${ }^{68}$ A. Nitzan and R. Silbey, J. Chem. Phys. 60, 4070 (1974).

${ }^{69}$ M. Bruehl and J. T. Hynes, Chem. Phys. 175, 205 (1993).

${ }^{70}$ I. Benjamin and R. M. Whitnell, Chem. Phys. Lett. 204, 45 (1993).

${ }^{71}$ M. Tuckerman and B. J. Berne, J. Chem. Phys. 98, 7301 (1993).

${ }^{72}$ S. Gnanakaran and R. M. Hochstrasser, J. Chem. Phys. 105, 3486 (1996).

${ }^{73}$ R. Rey and J. T. Hynes, J. Chem. Phys. 104, 2356 (1996).

${ }^{74}$ R. Larsen and R. M. Stratt, J. Chem. Phys. 110, 1036 (1999).

${ }^{75}$ S. A. Egorov and J. L. Skinner, J. Chem. Phys. 112, 275 (2000).

${ }^{76}$ K. F. Everitt, S. A. Egorov, and J. L. Skinner, Chem. Phys. 235, 115 (1998).

${ }^{77}$ K. F. Everitt and J. L. Skinner, J. Chem. Phys. 110, 4467 (1999).

${ }^{78}$ D. E. Sagnella et al., Biophys. J. 77, 70 (1999).

${ }^{79}$ J. L. Skinner and K. Park, J. Phys. Chem. B 105, 6716 (2001).

${ }^{80}$ J. Poulsen, S. R. Keiding, and P. J. Rossky, Chem. Phys. Lett. 336, 488 (2001).

${ }^{81}$ J. Poulsen and P. J. Rossky, J. Chem. Phys. 115, 8014 (2001).

${ }^{82}$ J. Poulsen and P. J. Rossky, J. Chem. Phys. 115, 8024 (2001).

${ }^{83}$ N. Makri, Annu. Rev. Phys. Chem. 50, 167 (1999).

${ }^{84}$ A. Nitzan, S. Mukamel, and J. Jortner, J. Chem. Phys. 60, 3929 (1974).

${ }^{85}$ A. Nitzan, S. Mukamel, and J. Jortner, J. Chem. Phys. 63, 200 (1975).

${ }^{86}$ D. C. Douglass, J. Chem. Phys. 35, 81 (1961).

${ }^{87}$ S. A. Adelman and R. H. Stote, J. Chem. Phys. 88, 4397 (1988).

${ }^{88}$ R. H. Stote and S. A. Adelman, J. Chem. Phys. 88, 4415 (1988).

${ }^{89}$ S. A. Adelman, R. Muralidhar, and R. H. Stote, J. Chem. Phys. 95, 2738 (1991).

${ }^{90}$ E. Rabani and D. R. Reichman, J. Phys. Chem. B 105, 6550 (2001).

${ }^{91}$ W. H. Press, B. P. Flannery, S. A. Teukolsky, and W. T. Vetterling, $\mathrm{Nu}$ merical Recipes (Cambridge University Press, Cambridge, 1986).

${ }^{92}$ D. Rostkier-Edelstein, P. Graf, and A. Nitzan, J. Chem. Phys. 107, 10470 (1997).

${ }^{93}$ D. Rostkier-Edelstein, P. Graf, and A. Nitzan, J. Chem. Phys. 108, 9598 (1998).

${ }^{94}$ K. F. Everitt, J. L. Skinner, and B. M. Ladanyi, J. Chem. Phys. 116, 179 (2002).

${ }^{95}$ P. H. Berens, S. R. White, and K. R. Wilson, J. Chem. Phys. 75, 515 (1981).

${ }^{96}$ L. Frommhold, Collision-Induced Absorption in Gases, Vol. 2, in Cambridge Monographs on Atomic, Molecular, and Chemical Physics, 1 st ed. (Cambridge University Press, England, 1993).

${ }^{97}$ J. L. Skinner, J. Chem. Phys. 107, 8717 (1997).

${ }^{98}$ S. C. An, C. J. Montrose, and T. A. Litovitz, J. Chem. Phys. 64, 3717 (1976).

${ }^{99}$ P. Schofield, Phys. Rev. Lett. 4, 239 (1960).

${ }^{100}$ P. A. Egelstaff, Adv. Phys. 11, 203 (1962).

${ }^{101}$ G. R. Kneller, Mol. Phys. 83, 63 (1994).

${ }^{102}$ J. Larsen et al., J. Chem. Phys. 116, 7997 (2002).

${ }^{103}$ E. Geva, Q. Shi, and G. A. Voth, J. Chem. Phys. 115, 9209 (2001).

${ }^{104}$ T. Yamamoto, J. Chem. Phys. 33, 281 (1960).

${ }^{105}$ W. H. Miller, J. Chem. Phys. 61, 1823 (1974)

${ }^{106}$ W. H. Miller, S. D. Schwartz, and J. W. Tromp, J. Chem. Phys. 79, 4889 (1983).

${ }^{107}$ W. H. Miller, J. Phys. Chem. A 102, 793 (1998).

${ }^{108}$ B. B. Laird, J. Budimir, and J. L. Skinner, J. Chem. Phys. 94, 4391 (1991). 